\title{
Development of a simulation model of HPPs chain operation
}

\author{
Alexandra Khalyasmaa ${ }^{1,2 *}$, Stanislav Eroshenko ${ }^{1,2}$, Sergey Mitrofanov $^{2}$, Anastasia $_{\text {Rusina }}^{2}$, Anna $_{\text {Arestova }}^{2}$, \\ Alena Sidorova $^{2}$ \\ ${ }^{1}$ Ural Federal University, Ural Power Engineering Institute, Ekaterinburg, Russia \\ ${ }^{2}$ Novosibirsk State Technical University, Faculty of Power Engineering, Novosibirsk, Russia
}

\begin{abstract}
The paper presents a simulation model of a hydroelectric power plants chain. The model allows solving the problem of hydro power plants (HPPs) operation mode planning in a unified power system, taking into account the optimization of water resources. The optimal filling and decrease of storage was performed in MATLAB Simulink software. The hydraulic properties of the river flow between the stations and the corresponding time lags in the functioning of the down-river station are taken into account. The model allows continuously monitoring changes in water pressure at hydropower plants and, as a result, uses the family of flow characteristics for various water pressures. The issues of optimizing the participation of hydroelectric power stations in the regimes of large hydrothermal power systems were also raised.
\end{abstract}

\section{Introduction}

The world is increasingly focusing on renewable energy [1]. It is necessary to create mathematical models to verify the principles of control during their functioning in electric power systems (EPS) [2]. Models of "traditional" renewable sources - hydroelectric power plants (HPPs) also need to be adapted. The relevance of such developments is increasing for HPPs operating in the cascade [3]. It is known that the power of a hydroelectric power plant and a cascade as a whole depends on water flow rate and pressure.

Traditionally, consumable characteristics can be obtained in the following ways: according to the recommendations of the manufacturer from the equipment certificate, according to the full-scale tests, based on the data of the automated control system.

Changes in characteristics can be influenced by constant factors (state of the turbine setting, stator, rotor, control system adjustment, technical condition of the units, hydraulic turbines wear from cavitation, etc.) and by variable factors (unevenness of the water flow, clogging of the trash racks, unsteady regimes in the downstream and hydroturbine, cavitation processes) [4].

Due to the complexity of the process, updating the flow characteristics is rarely enough. However, academic papers appear where studies are carried out to manage the above-listed factors in challenges of optimal load distribution both inside the HPPs and between the plants. For example, in [5], a model of units' status records is listed using a model based on fuzzy logic.

In the tasks of optimizing long-term regimes, an adaptive simulation model can also be created that allows updating the water pressure in accordance with the predicted value of the natural inflow. Then the static calculation of the generated power of the HPPs (according to a single characteristic) can be represented as a series of characteristics with a volatile operating point (Figure 1). The operating point will depend on the water pressure. The adjusted water flow rate will make it possible to more accurately optimize the loading of the HPPs cascade.

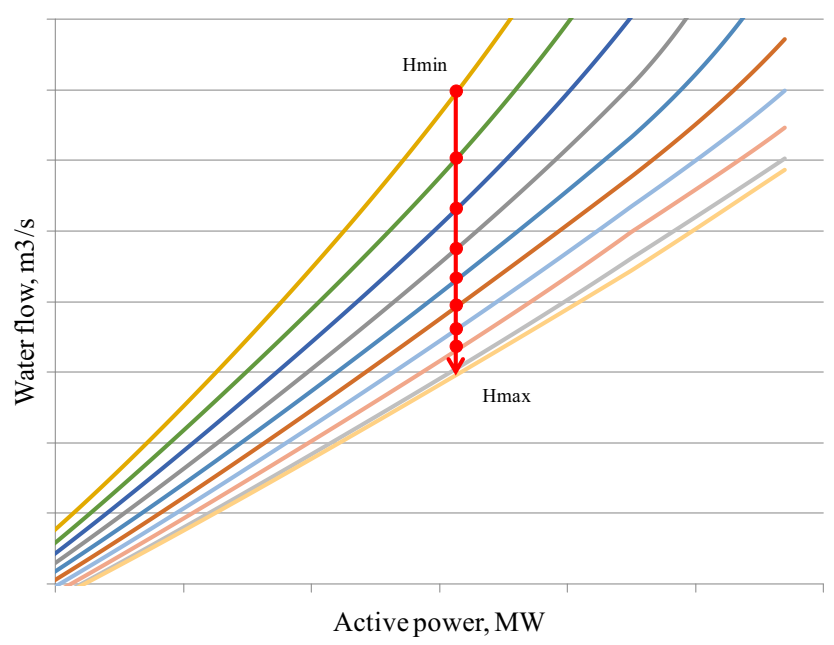

Fig 1. Discharge characteristics of hydroelectric unit

It should be noted that the model presented in the paper is one of the stages in the implementation of the authors' idea of creating tools that allow improvement of the general EPS performance [6].

\section{Mathematical model}

The principle of the HPPs cascade operation in the EPS can be represented as a mathematical model with a specific algorithm, which is subject to well-time

Corresponding author: lkhalyasmaa@mail.ru 
correction. An important aspect of process modeling of a real HPP is to take into account the unpredictability and variability of runoff, which depends on the nourishments of the river, meteorological factors, everyday afflux, etc. If we consider only the large rivers of Russia, then the benchmarks of volatility vary from $1 \%$ to $50 \%$ and the period - from day to year [7]. To account for such significant indicators, a special algorithm is required, which includes informational support for the water runoff, runoff models, simulation methods, adaptive schemes, statistical analysis as well as an interactive decision-making system.

What follows is a detailed description of the elaborated simulation model of the HPP cascade. The simulated cascade consists of two hydroelectric power plants. A general view of the model is shown in Figure 2. A hydropower plant overlying the river is designated as HPP No. 1, and the underlying is HPP No. 2. The operation algorithms of each of the plants are presented in Figure 3.

The model contains two information modules:

1. "Electricity" - the load profile of the EPS, which must be ensured by generating a cascade of HPPs;

2. "Water" - a graph of the natural inflow of water, followed by withdrawal of water for various needs.

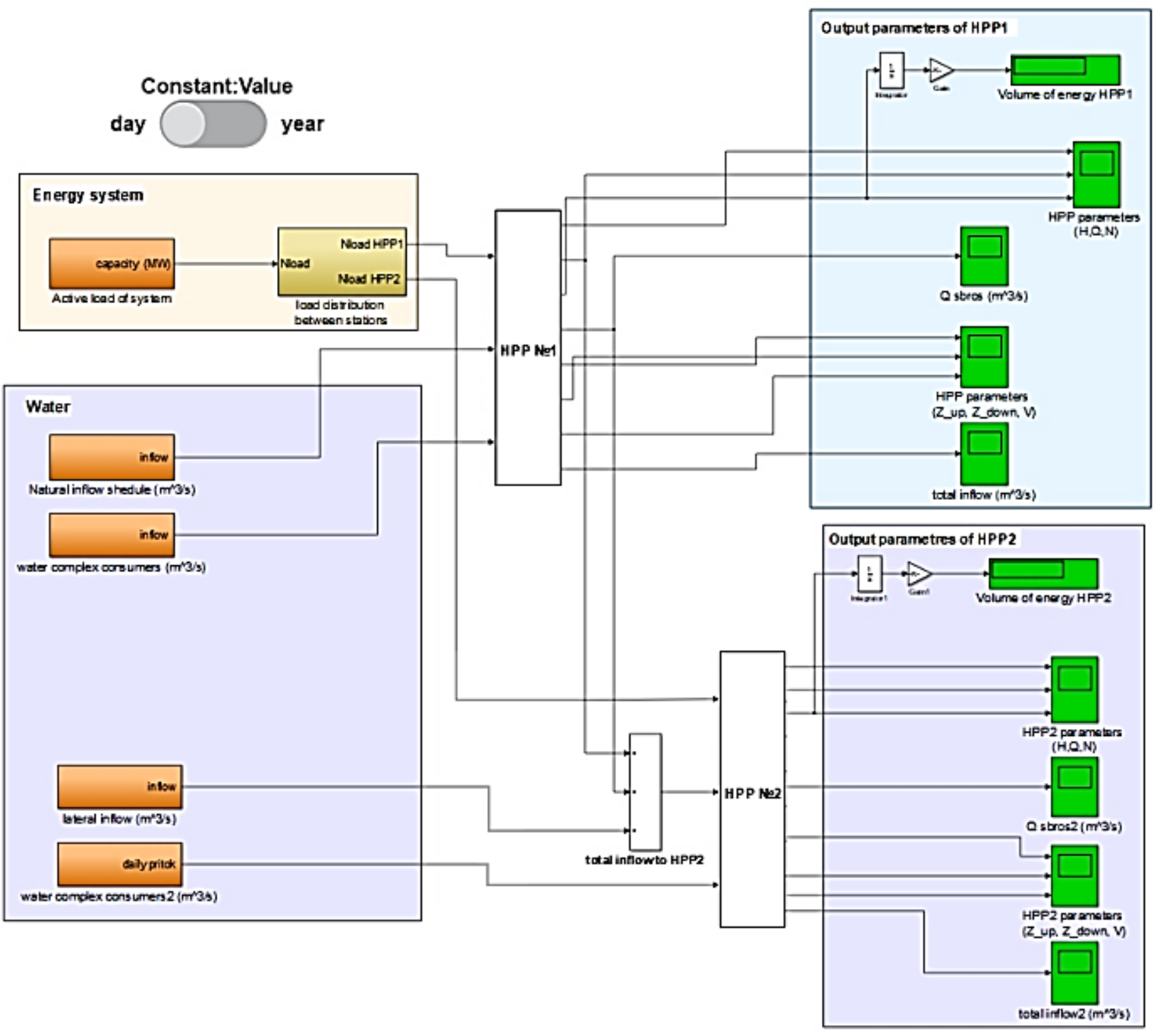

Fig 2. Model of the HPP cascade in Matlab Simulink

The initial data for the "electricity" module (Figure 3) is represented by the "active load of system" block (1). The features of hydrological processes are taken into account in the blocks: "natural inflow schedule" (2), "water complex consumers" (3), “filtration" (5), "evaporation" and "ice formation" (6).

Hereafter, links to the blocks indicated by numbers in Figures 2 and 3 are given in parentheses in the text. 


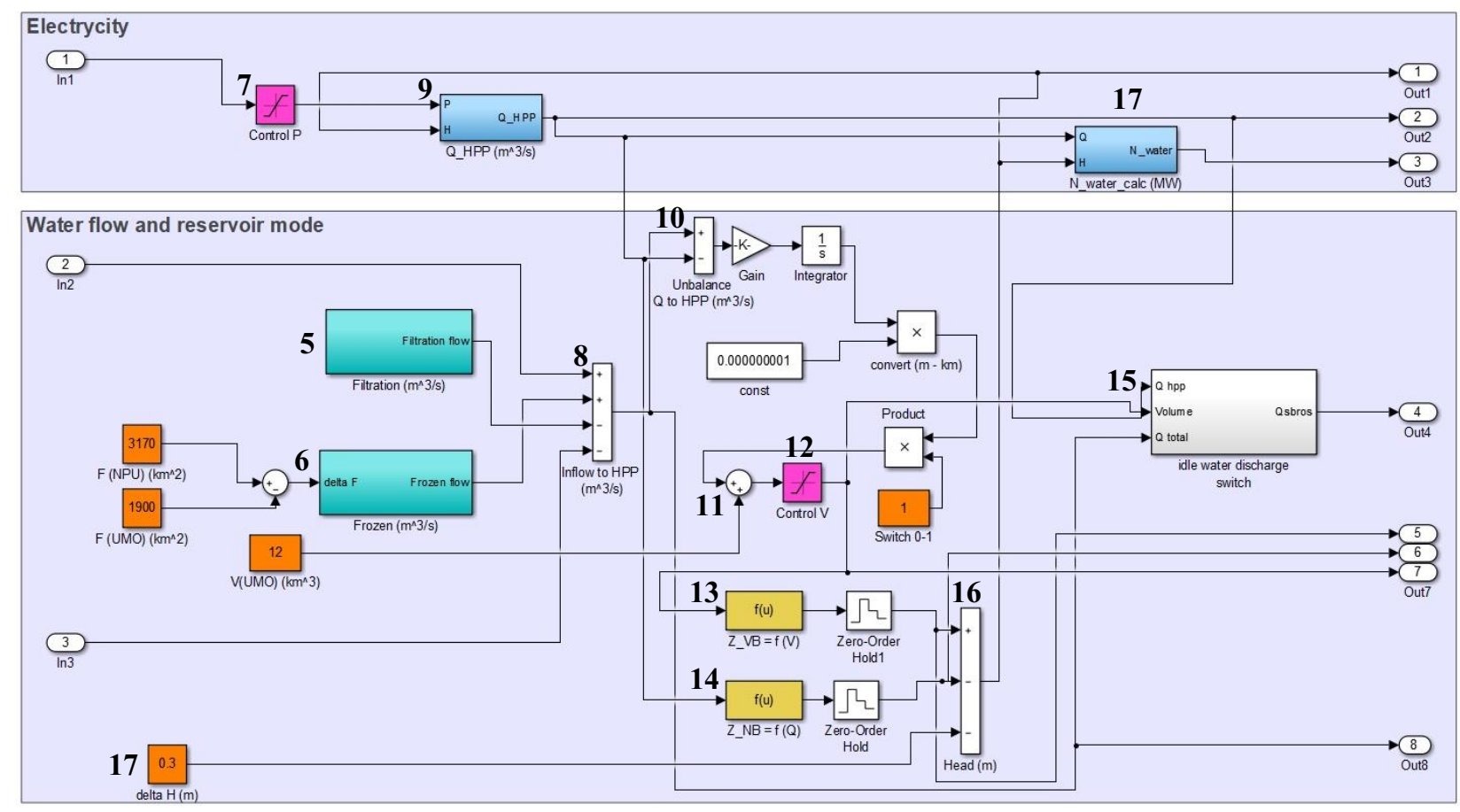

Fig 3. Operation algorithms of HPP cascade

\subsection{Module "Electricity"}

In the case of the operation of both plants of the cascade in a single electric network, it is required to determine the participation coefficient of each plant in the load schedule coverage. The optimization of the share of each plant block is designated as "load distribution between plants" (4). The load between plants in this block is distributed according to the condition:

$$
\frac{\partial Q_{H P P}}{\partial P_{H P P}}=\mathrm{idem}
$$

A number of restrictions are imposed on the share of the active capacity of HPPs. Firstly, there are requirements of the water management complex (WMC) to provide water to downstream consumers. From the point of view of efficient use of water resources, it is necessary to guarantee this volume of water in the downstream by passing it through the HPP turbines, and not by escapage [8]. Therefore, there is a limitation on the minimum capacity of HPP.

Secondly, the load power cannot exceed the installed capacity of the hydroelectric power station [9]. The control of two constraints is carried out by the "control P" block (7).

$$
P_{M I N . \mathrm{WMC}} \leq P_{\mathrm{SYS}} \leq P_{\mathrm{INSTL} . \mathrm{HPP}}
$$

\subsection{Module "Water"}

The "natural inflow schedule" block (2) sets the graph of the unevenness of the natural flow of water to the site of the overlying HPP. Accurate calculation and forecast of natural inflow increase the efficiency of using the HPP cascade. The problem of natural inflows forecasting was not considered herewithin.

The useful inflow of water fed to the turbines of the HPP can be obtained by taking into account the flow of water to consumers of the water complex consumers «water complex consumers» (3), filtering through the dam base "filtration" (5) and ice formation "ice formation" (6). In this model, the assumption is made that the water consumption for evaporation is insignificant in comparison with other variables, and is not included in the calculation. The resulting inflow to the HPP site is calculated in the "inflow to HPP" block (8).

$$
Q_{\text {RES.INFLOW }}=Q_{I N F L O W}-Q_{F I L T}-Q_{W M C} \pm Q_{I C E}
$$

The calculation of the water flow corresponding to the required active power is in the block "Q_HPP" (9):

$$
Q_{P_{H P P}}=\frac{P_{\mathrm{LOAD}}}{9,81 \eta H},
$$

where: $\eta=0,94$ - unit efficiency, $H$ - pressure.

Excess water not flowing through the turbines of the HPP is accumulated in the reservoir.

$$
Q_{\text {RESERVOIR }}=Q_{\text {RES.INFLOW }}-Q_{\text {TURB }}
$$

The volume of water in the reservoir is determined based on the following:

$$
V_{\text {RESERVOIR }}=V_{\text {RESERVOIR }}(\mathrm{DSL}) \pm \int Q_{\text {RESERVOIR }} d t,
$$

where: $V_{\text {RESERVOR }}(\mathrm{DSL})$ - volume of water in the reservoir at the dead storage level, $Q_{\text {RESERVOIR }}$ - water flow from the reservoir. 
One of the main limitations for the reservoir operation mode is the restriction on the levels of DSL and NWL (normal water level):

$$
Z_{\text {UPSTR }}(\mathrm{DSL}) \leq Z_{\mathrm{UPSTR}} \leq Z_{\mathrm{UPSTR}}(\mathrm{NWL})
$$

This restriction can be set through the values of the reservoir volume [10]:

$$
V_{\text {RESERVOIR }}(\mathrm{DSL}) \leq V_{\text {RESERVOIR }} \leq V_{\text {RESERVOIR }}(\mathrm{NWL})(8)
$$

This condition is monitored by the control V block.

The upstream level directly depends on the volume of water in the reservoir. The relationship of these parameters is determined by the characteristic:

$$
Z_{\text {UPSTR }}=f\left(V_{\text {RESERVOIR }}\right)
$$

This function is modeled in the block "Z_VB $=\mathrm{f}$ (V)" and is shown in Figure 4.

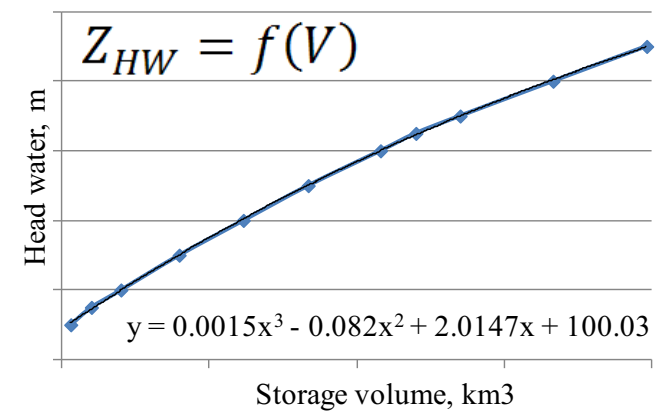

Fig 4. The curve of the upstream level versus the volume of water.

The water level in the downstream depends on the flow rate passing through the site of the HPP. Downstream consumption is determined by the following equation:

$$
Q_{\text {DOWNTR }}(t)=Q_{T U R B}(t)+Q_{E S C P}(t),
$$

where: $Q_{\text {TURB }}$ - flow rate through HPP turbines, $Q_{E S C P}$ - discharge through spillway structures.

The relationship of these parameters is determined by the characteristic:

$$
Z_{\text {DOWNSTR }}=f\left(Q_{\text {DOWNSTR }}\right)
$$

This function is modeled in the block "Z_NB $=\mathrm{f}$ (Q)" and is shown in Figure 5.

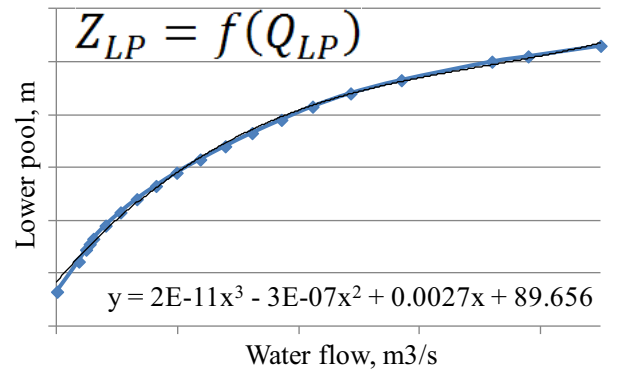

Fig 5. Downstream level versus. water flow curve.
The expense balance is represented by the following mathematical model [11]:

$$
\begin{gathered}
Q_{\text {INFLOW }}(t) \pm Q_{\text {RESERVOIR }}(t) \pm Q_{\text {FILTER }}(t) \pm \\
\pm Q_{\text {ICE }}(t)-Q_{W M C}(t)=Q_{T U R B}(t)+Q_{E S C P}(t),
\end{gathered}
$$

where: $Q_{\text {INFLOw }}$ - natural inflow, $Q_{\text {RESERVIR }}$ - water flow from the reservoir, $Q_{\text {FILTER }}$ - seepage discharge, $Q_{I C E}$ - consumption in the ice formation and melting in the upstream dam, $Q_{W M C}-\mathrm{WMC}$ requirements.

If the reservoir volume reaches its maximum value, escapage occurs. Escapage at the station is an extremely undesirable phenomenon, since it leads to irrational use of water resources and lost profits. However, it is very difficult to avoid during the flood period [12].

The condition for the start of escapage is:

$$
\left(Z_{\mathrm{UPSTR}} \approx N W L\right) \cup\left(Q_{\mathrm{TURB}}<Q_{\mathrm{INFLOW}}\right)
$$

In the model presented here, the period of the start of escapage is determined by the achievement of the upstream level of the NWL mark. Moreover, the escapage value is determined by the following:

$$
\begin{gathered}
Q_{\text {ESCP }}(t)=Q_{\mathrm{TURB}}(t)-Q_{\text {INFOW }}(t) \pm Q_{\text {RESERVOIR }}(t) \pm \\
\pm Q_{\text {FILTER }}(t) \pm Q_{\mathrm{ICE}}(t)-Q_{W M C}(t) .
\end{gathered}
$$

The pressure of a HPP is defined as the difference between the level of the upstream and downstream taking into account losses in water supply facilities:

$$
H=Z_{U P S T R}-Z_{\text {DOWNSTR }}-\Delta h,
$$

where: $\Delta h$ - pressure losses in water supply facilities - “delta H” block.

As well as for other basic parameters, there is a block for pressure that is responsible for its control.

The value of the flow rate and the changing pressure are fed to the "N_water_calc" block, where the total generated power of the HPP is calculated.

The model of the underlying station has slight differences from HPP No. 1. The difference at this stage will be the calculation of the natural inflow to the HPP site No. 2. The inflow is formed on the basis of the values of the water flow to the HPP No 1 downstream and lateral inflow in the area between plants:

$$
Q_{\text {NAT.INFLOW }}=Q_{\mathrm{HPP} 1}+Q_{\mathrm{LAT.INFLOW}}
$$

\section{Simulation results}

Figures 6-7 show the simulation results of HPP cascade with a daily regulation reservoir. Figure 6 shows a graph of the active power load of the HPP in the daily range and the water flow rate that is required for its production taking into account the changing water pressure. Figure 7 shows the levels of the upstream and downstream, representing the filling and discharge of the reservoir. 


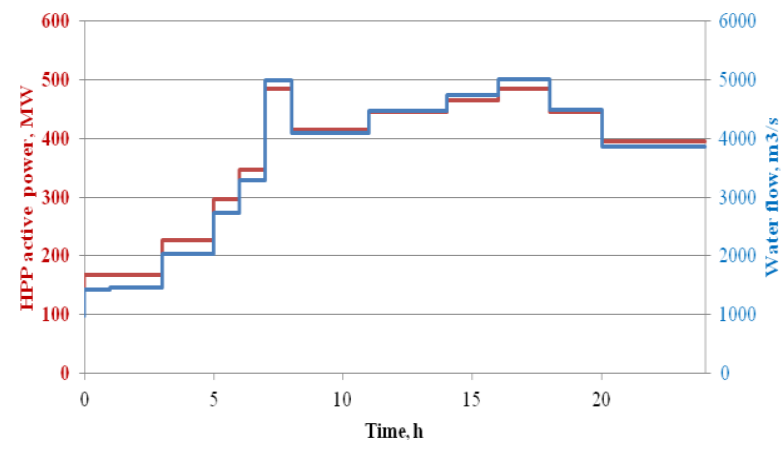

Fig 6. Daily schedule for changes in the active power of hydroelectric power generation and the water flow

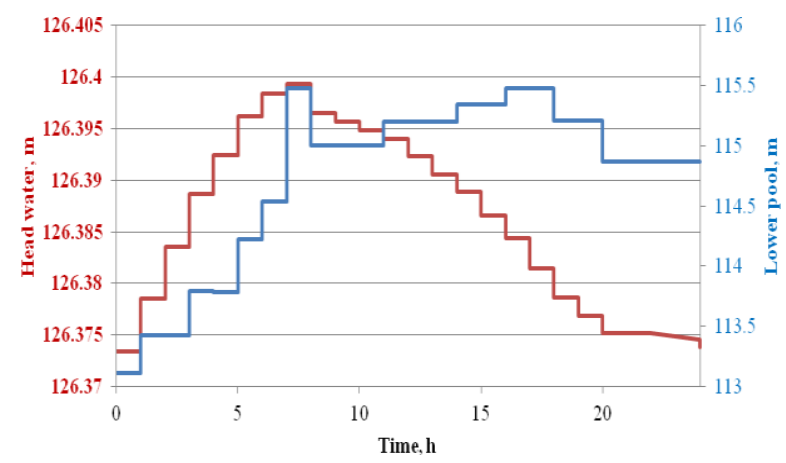

Fig 7. Schedule of changes in the levels of the upstream and downstream of the reservoir in the daily regulation range

To obtain reliable simulation results of hydraulic modes, it is necessary to have fairly accurate initial data: curves of changes in the levels of the upstream and downstream, the slope of the river channel, the area of the reservoir surface, the flow rate of the river, and much more. As a rule, the reliability and sufficiency of the initial data for existing HPP is extremely low. Prediction and calculation of input parameters for the simulation model can be based on spatial data of geographic information systems (parameters of hydrography, snow cover, surface temperature, topography, etc.). Details of the use of spatial data for the simulation model will be presented in future publications.

The proposed model can be used for the following:

1. To increase the controllability and energy efficiency of generating electric power through the HPPs cascade.

2. To prove the need to take into account the mutual influence of electric and hydraulic modes of operation of the power system with the participation of HPPs in order to increase the efficiency of its management.

3. To take into account the restrictions of the underlying and overlying stations located on the same river.

4. For the economic justification for the construction of hydropower plants.

5. In addition, the presented model can become the basis for creating a prototype model of the power system with included HPP cascades

\section{Conclusion}

The simulation model allows to calculate the modes of electric power systems with a HPP cascade with any lead time and takes into account the characteristic aspects of their technological processes.

The algorithms implemented in the simulation model can be prototypes of the algorithms for group regulation of active power, can be used to control the modes of HPPs and their cascades, and a series of calculations will simplify the decision-making mechanism by the staff.

The elaborated simulation model allows monitoring the operating parameters of EPS with a HPP cascade, which ensures optimal use of water resources and increase the efficiency of the EPS as a whole.

\section{References}

1. T. Mai et al., "Renewable Electricity Futures for the United States," in IEEE Transactions on Sustainable Energy, vol. 5, no. 2, pp. 372-378, April 2014, doi: 10.1109/TSTE.2013.2290472.

2. H. Xiaoyun, H. Andi and S. Jingpeng, "Short-term Hydro Power Scheduling under the Electricity Market Environment," 2019 2nd International Conference on Safety Produce Informatization (IICSPI), Chongqing, China, 2019, pp. 473-477, doi: 10.1109/IICSPI48186.2019.9095949.

3. B. Tong, X. Guan, Q. Zhai and F. Gao, "Long-term scheduling of cascaded hydro energy system with distributed water usage allocation constraints," 2011 IEEE Power and Energy Society General Meeting, Detroit, MI, USA, 2011, pp. 1-7, doi: 10.1109/PES.2011.6039225.

4. Fan-nie Kong and Yan Li, "Cultural algorithm based long-term optimization scheduling of cascaded Hydro-Plant," 2011 IEEE International Conference on Computer Science and Automation Engineering, Shanghai, 2011, pp. 136-139, doi: 10.1109/CSAE.2011.5953187.

5. Y. Sekretarev. Investigations of possible using a generalized fuzzy interval for analyzing operating conditions of power equipment at hydropower plants. IEEE 3.26, 6 (2016)

6. Composite author. HPP: the art of control engineering. Monograph. NSTU Publisher (2019)

7. T. Filippova. Hydroelectricity. Tutorial. NSTU Publisher (2012)

8. P. Borshch. Methods of planning the generation of electricity in a hydroelectric power plants cascade, taking into account draining and atmospheric factors. Thesis of technical sciences candidate (2014)

9. C. Le. Control of a hydroelectric power plants cascade. News of the Southern Federal University. Technical science 15, 24-32 (2006)

10. P. Erokhin. Optimization of long-term operation modes of hydropower plants by the criterion of minimizing the costs of electricity consumers on 
the Russian wholesale market. Energetic 11, 39-41 (2013)

11. A. Rusina. Tasks of optimal performance of hydroelectric in power system. IFOST, Novosibirsk, 2, 251-254 (2016)
12. S. Mitrofanov. Simulation model of cascaded HPPs in Pamir. IFOST, China, Harbin, IEEE, 889-892 (2018) 